(2) Open Access Full Text Article

REVIEW

\title{
Walking impairment in patients with multiple sclerosis - a new therapeutic approach and clinical potential of dalfampridine extended release tablets
}

This article was published in the following Dove Press journal:

Degenerative Neurological and Neuromuscular Disease

22 June 2012

Number of times this article has been viewed

\author{
Herbert R Henney III \\ Andrew R Blight
}

Acorda Therapeutics, Inc, Hawthorne, NY, USA
Correspondence: Herbert R Henney III Acorda Therapeutics, Inc, I5 Skyline Drive, Hawthorne, New York 10532, USA

$\mathrm{Tel}+\mid$ 9|4 3474300 ext 4 I 38

$\mathrm{Fax}+$ I 9143474560

Email hhenney@acorda.com

\begin{abstract}
Walking impairment is a clinical hallmark of multiple sclerosis (MS) that has been under-recognized as a therapeutic target for pharmacologic intervention. The development and approval of dalfampridine extended release tablets (dalfampridine-ER; known as prolonged-, modified, or sustained-release fampridine outside the USA), $10 \mathrm{mg}$ taken twice daily, to improve walking in patients with MS, fills a previously unmet need. In three randomized, double-blind, placebo-controlled trials, dalfampridine-ER improved walking speed in approximately onethird $(37 \%)$ of treated patients, and average walking speed on therapy among these responders improved by approximately $25 \%$ relative to baseline. Walking-speed improvement among responders was clinically significant, as determined by a statistically significant improvement in the patient-reported 12-item Multiple Sclerosis Walking Scale. Long-term extension studies indicate that responders were able to maintain benefits, compared with nonresponders over prolonged periods of treatment. Dalfampridine-ER was generally well tolerated. Dizziness, insomnia, balance disorder, headache, nausea, urinary tract infection, and asthenia were the most common adverse events. Although the incidence of seizures appeared to be dose related, among patients treated with dalfampridine-ER in the three trials, the rate of seizures was $0.25 \%$. These efficacy and safety data suggest that dalfampridine-ER can be a useful and clinically relevant addition to the pharmacologic armamentarium for the management of MS symptoms and disabilities. Because of its narrow therapeutic index and potential for seizures, it is especially important in the clinical setting to adhere to the dosing recommended in the approved labels.
\end{abstract}

Keywords: multiple sclerosis, walking, mobility, dalfampridine, fampridine, 4-aminopyridine

\section{Introduction}

Multiple sclerosis (MS) is a chronic disease with onset usually occurring by the fourth decade of life and a progressive course of disability over a generally normal life expectancy. As of 2011, the estimated prevalence was approximately 400,000 individuals in the USA and more than 2.5 million worldwide. ${ }^{1}$ Because of its early onset and long duration, it is associated with a disproportionate socioeconomic burden, relative to more common medical conditions, which has been estimated to exceed US\$2 million over the lifetime of an individual. ${ }^{2}$ This burden is related to the direct medical costs associated with long-term management and to indirect and intangible costs resulting from the impairment of function, which has a substantial impact on patients' daily lives. In particular, this impact includes a lower quality of life relative to the general population; ${ }^{3,4}$ reduced productivity and earnings,${ }^{5}$ which have been suggested as 
the greatest contributing factors to the societal burden of $\mathrm{MS} ; 3,4$ and the need for adaptive equipment in the home or workplace, as well as formal and informal care. ${ }^{2}$ Importantly, this caregiving is itself associated with a caregiver burden, the magnitude of which may be dependent on the specific symptoms present in the patient with MS. ${ }^{6-8}$

The pathophysiology of MS is characterized by inflammatory demyelination and degeneration of axons within the central nervous system. ${ }^{9-11}$ Current MS management strategies rely on immunomodulatory drugs that target inflammatory processes with the goal of reducing relapses and delaying disability progression. Demyelination is a wellrecognized pathologic characteristic of MS that is responsible for a delay or blocking of action-potential conduction that produces much of the neurologic deficits and disabilities characteristic of MS, including walking impairment. ${ }^{10,12,13}$

Among the disabilities associated with MS, walking impairment is a hallmark symptom that is also important from the perspective of clinical diagnosis, since it serves as a primary indicator of disease progression, as generally measured by the Expanded Disability Status Scale (EDSS) and the Multiple Sclerosis Functional Composite (MSFC). ${ }^{14,15}$ The EDSS is a clinician-rated scale that ranges from 0 , representing a normal neurologic exam, to 10, representing death due to MS; ${ }^{14}$ the MSFC enables clinical assessment of disability based on measurement of walking speed, arm dexterity, and cognitive function. ${ }^{15}$

In addition to the clinical perspective, walking impairment is highly relevant from the patient's perspective, since it affects mobility, function, independence, and quality of life. ${ }^{5,16-18}$ In a study by Heesen et al, ${ }^{19}$ lower-limb function was ranked of highest importance among 13 bodily functions by the greatest proportion of patients regardless of level of disability and disease duration. Additionally, in a survey of 1011 individuals with MS, 70\% of those who experienced difficulty walking reported that it was the biggest challenge associated with their disease. ${ }^{18}$

Although a longitudinal study estimated that the probability of requiring walking assistance 15 years after diagnosis was approximately $40 \%$ and of requiring a wheelchair $25 \%,{ }^{20}$ walking impairment is evident early in the disease process, often with concomitant balance impairment. ${ }^{21}$ Even among patients with otherwise low levels of disability, walking impairment was manifested as reductions in both speed and distance relative to healthy controls among patients with EDSS scores as low as 0-2.5.22 More recently, in a survey of 436 patients with MS from the United Kingdom, France, Germany, Sweden, Spain, and Canada, almost half the patients $(45 \%)$ reported experiencing mobility difficulties within a month of diagnosis, and $93 \%$ of patients reported difficulties within 10 years. $^{23}$

For many of the symptoms and disabilities present in patients with MS, pharmacologic therapies have been available and are recommended as part of a comprehensive approach to disease management. ${ }^{24-27}$ While assistive devices and various forms of physical therapy have been used to ameliorate the effects of walking impairment, only recently has the first pharmacologic therapy specifically targeting this disability become available. In January 2010, dalfampridine extended release tablets (dalfampridine-ER [AMPYRA ${ }^{\circledR}$ ]; prolonged-, modified, or sustained-release fampridine [FAMPYRA ${ }^{\circledR}$ ] in some countries), $10 \mathrm{mg}$ to be administered twice daily approximately 12 hours apart, were approved by the US Food and Drug Administration. ${ }^{28}$ This was the first drug indicated to improve walking in patients with MS. It is available outside the US, where it is known by its previous US-adopted name and current international nonproprietary name, fampridine. ${ }^{29,30}$

\section{Dalfampridine-ER Development}

Dalfampridine-ER, chemically 4-aminopyridine (4-AP), is a broad-spectrum potassium channel blocker that has been used as an in vitro research tool in electrophysiologic studies and for characterizing the structure and function of potassium channels. Its putative mechanism of action in MS is the restoration of conduction via blockade of the potassium channels that become exposed during demyelination; ${ }^{31}$ this restoration of conduction is mainly a result of its ability to enhance the propagation of the impulses across demyelinated axonal segments. ${ }^{32,33}$

Formal clinical development of dalfampridine-ER for walking impairment was pursued based on early studies of intravenous or immediate-release oral formulations that suggested benefits in motor function in patients with MS. ${ }^{34-36}$ These effects were confirmed in a small study $(\mathrm{N}=10)$ using a sustained-release formulation that showed significant improvements in gait speed relative to placebo. ${ }^{37}$ The need for an extended release formulation was based on the narrow therapeutic range and relatively short half-life of dalfampridine.

\section{Pharmacokinetic characteristics}

An excretion-balance study demonstrated that elimination of an orally administered solution of $15 \mathrm{mg}{ }^{14} \mathrm{C}$-radiolabeled 4-AP was rapid and complete, with recovery of $96.4 \%$ in urine by 24 hours, primarily as unchanged compound, and 
only $0.5 \%$ recovered in feces.$^{38}$ However, immediate-release formulations are characterized by rapid and high peak-serum levels associated with an increased risk of adverse events (AEs) ${ }^{39,40}$ The goal of developing the extended release tablets was to optimize the pharmacokinetic characteristics by maintaining therapeutic exposure while reducing the maximum plasma concentration $\left(\mathrm{C}_{\max }\right)$ and increasing the time to $\mathrm{C}_{\max }\left(\mathrm{t}_{\max }\right)$. As shown in Table 1, administration of the immediate-release oral formulation at a therapeutic dose of $10 \mathrm{mg}$ is rapidly absorbed with a $\mathrm{t}_{\max }$ of 1.0-1.2 hours in healthy volunteers and a $\mathrm{C}_{\max }$ of $46.4 \pm 9.7 \mathrm{ng} / \mathrm{mL}{ }^{41}$ In contrast, $10 \mathrm{mg}$ tablets of dalfampridine-ER result in a $\mathrm{C}_{\max }$ that is approximately half that of the immediate-release formulation, with a $t_{\max }$ and apparent half-life $\left(t_{1 / 2}\right)$ that are both twice that of immediate release and are comparable in healthy volunteers and in patients with MS (Table 1). ${ }^{42,43}$ Similar pharmacokinetics were observed under steady-state conditions: dalfampridine-ER at $10 \mathrm{mg}$ twice daily resulted in a $t_{\max }$ of 3.9 hours, $\mathrm{a}_{\text {max }}$ of $25.3 \mathrm{ng} / \mathrm{mL}$, and an area under the curve (AUC) of $200 \mathrm{ng} \cdot \mathrm{h} / \mathrm{mL} .{ }^{44}$ The pharmacokinetics of dalfampridine-ER were studied in healthy volunteers under fed and fasted conditions. The differences between the fed and fasted states were found to be not clinically relevant based on geometric means of $\mathrm{C}_{\text {max }}$ (fed, $27.4 \mathrm{ng} / \mathrm{mL}$; fasted $22.3 \mathrm{ng} / \mathrm{mL}$ ) and $\mathrm{AUC}_{0-\infty}$ (fed, $244.1 \mathrm{ng} \cdot \mathrm{h} / \mathrm{mL}$; fasted, $248.9 \mathrm{ng} \cdot \mathrm{h} / \mathrm{mL}$ ). Although slowing the absorption phase by $23 \%$, food had minimal influence on the extent of dalfampridine-ER absorption, suggesting administration without regard to food intake. ${ }^{45}$

The more favorable pharmacokinetic profile of dalfampridine-ER is consistent with twice daily dosing, which provides an additional advantage over the four-times daily dosing that would be required with immediate release; less frequent dosing has been suggested to result not only in greater patient adherence, ${ }^{46,47}$ but also in improving outcomes and reducing costs. ${ }^{48}$

Table I Mean ( \pm standard deviation) single-dose pharmacokinetic parameters of immediate-release formulations of 4-aminopyridine (4-AP) and dalfampridine extended release $10 \mathrm{mg}$ tablets

\begin{tabular}{lccr}
\hline $\begin{array}{l}\text { Pharmacokinetic } \\
\text { parameter }\end{array}$ & $\begin{array}{l}\text { Immediate- } \\
\text { release } \\
\text { 4-AP I0 } \mathbf{~ m g} \\
(\mathbf{N}=\mathbf{6})^{41}\end{array}$ & $\begin{array}{l}\text { Dalfampridine extended } \\
\text { release 10 } \mathbf{~ m g ~}\end{array}$ \\
\cline { 3 - 4 } & $\begin{array}{l}\text { Healthy } \\
\text { volunteers } \\
(\mathbf{N}=\mathbf{5})^{42}\end{array}$ & $\begin{array}{l}\text { Patients } \\
\text { with } \mathbf{M S} \\
\mathbf{( N = 2 4 )}\end{array}$ \\
\hline $\mathrm{t}_{\text {max }}, \mathrm{h}$ & $1.2 \pm 0.4$ & $3.2 \pm 1.5$ & $3.9 \pm 1.2$ \\
$\mathrm{C}_{\max }, \mathrm{ng} / \mathrm{mL}$ & $46.4 \pm 9.7$ & $21.6 \pm 3.89$ & $25.2 \pm 6.8$ \\
$\mathrm{t}_{1 / 2}, \mathrm{~h}$ & $3.7 \pm 0.7$ & $6.4 \pm 1.3 \mathrm{I}$ & $5.6 \pm 2.2$ \\
AUC $_{0-\infty}, \mathrm{ng} \cdot \mathrm{h} / \mathrm{mL}$ & $184.6 \pm 24.0$ & $284.8 \pm 31.78$ & $283.2 \pm 88.2$ \\
\hline
\end{tabular}

Abbreviations: AUC, area under the curve; MS, multiple sclerosis.

\section{Phase II and Phase III clinical trials of dalfampridine-ER}

Approval of dalfampridine-ER was based on pivotal clinical trials that demonstrated significant improvements relative to placebo in walking speed and patient-reported improvement of walking, and a generally favorable tolerability profile. ${ }^{49,50}$ In these studies, walking speed was assessed using the Timed 25-Foot Walk (T25FW), a component of the MSFC..$^{51}$ The 12-Item Multiple Sclerosis Walking Scale (MSWS-12) ${ }^{52}$ was used to provide clinical relevance from the patient's perspective; the MSWS-12 is a validated patient-reported measure that assesses the impact of MS on walking ability, with a higher score indicating greater difficulty walking.

In addition to the pivotal trials, dalfampridine-ER $10 \mathrm{mg}$ twice daily was used in long-term, open-label extensions of the trials. Because it has been available on the US market since March 2010, postmarketing data have been collected on its safety and tolerability in the clinical-practice setting.

Dalfampridine-ER was evaluated in one Phase II (MS-F202) and two Phase III (MS-F203 and MS-F204) clinical trials, of which the Phase III trials represent the pivotal studies for the demonstration of tolerability and efficacy for walking improvement. Assessment of walking during the dalfampridine-ER clinical development program utilized the $\mathrm{T} 25 \mathrm{FW}$ as the primary outcome measure. Although there are a variety of measures to assess walking and mobility in patients with $\mathrm{MS},{ }^{53}$ there exists a strong and clinically relevant rationale for use of the $\mathrm{T} 25 \mathrm{FW}$, including the observations that timed-walk speed correlates well with walking over longer distances and times. ${ }^{54}$ The T25FW is one of the three components of the MSFC, and has been found to be a valid and reliable measure over time, with negligible practice effects. ${ }^{55,56}$ The $\mathrm{T} 25 \mathrm{FW}$ also shows a moderate-to-strong correlation with the EDSS across MS types and level of walking impairment, ${ }^{51,57}$ and a change of $20 \%$ was identified as the minimum change that could be considered clinically meaningful. ${ }^{58-60}$ Furthermore, since the T25FW requires a minimum of time and space, this measure has been suggested to be practical in the clinical setting, ${ }^{53}$ and it has subsequently been recommended as a preferred measure, since it adequately describes walking capacity in patients with MS. ${ }^{61}$

The Phase II trial was a multicenter, randomized, doubleblind, placebo-controlled, parallel-group dose-ranging study that evaluated the efficacy of 10,15 , and $20 \mathrm{mg}$ dalfampridineER twice daily in patients with MS $(\mathrm{N}=206) .{ }^{62}$ The primary endpoint was percent change in walking speed on the T25FW. Although all three dalfampridine-ER dose groups showed 
average increases in walking speed that were numerically greater than placebo, none of the differences was statistically significant.

In a post hoc analysis of these data, a subset of patients was identified in each dose group who had consistently faster walking speeds during treatment assessments relative to off-treatment assessments, with the additional observation that there were more of these responders in the pooled dalfampridine-ER group, compared with placebo $(36.7 \%$ vs $8.5 \% ; P<0.05) .{ }^{62}$ Interestingly, no dose-response effect was observed; all doses used in the study resulted in a similar response rate $(35.3 \%-38.6 \%)$. These responders demonstrated a mean improvement of $25 \%$ to $29 \%$ in walking speed, exceeding the value of the $20 \%$ change that is considered clinically relevant. ${ }^{59,63-65}$

For this post hoc analysis, a responder was defined as a patient with a faster walking speed for at least three of the four visits during the double-blind treatment period, compared with the maximum speed for any of the five offdrug visits. This responder definition was subsequently used as a prospective criterion in the Phase III clinical trials.

The two pivotal Phase III trials prospectively incorporated the responder criterion into the primary efficacy endpoint, which was the percent of consistent timed-walk responders. ${ }^{49,50}$ Both trials were of similar double-blind, placebo-controlled design, and evaluated the $10 \mathrm{mg}$ twice daily dose relative to placebo over treatment periods of 14 weeks (MS-F203; $\mathrm{N}=301$, randomized) and 9 weeks (MS-F204; $\mathrm{N}=239$, randomized). In addition to the primary endpoint of objectively measured walking speed, the MSWS-12 was used to evaluate changes in walking from the patient's perspective in order to establish the clinical meaningfulness of the response.

In both pivotal trials, the proportion of timed-walk responders in the dalfampridine-ER group was higher than in the placebo group. In addition to these objective improvements, benefits in walking ability were achieved from the patient's perspective among timed-walk responders in both studies, independent of treatment assignment. In MS-F203, the average change from baseline in MSWS-12 score during the treatment period was $-6.84(95 \% \mathrm{CI},-9.65$ to -4.02$)$ for timed-walk responders and 0.05 (95\% CI, -1.48 to 1.57) for nonresponders, independent of treatment assignment $(P=0.0002)$. Comparable results were obtained in MS-F204; the average change from baseline was -6.04 (95\% CI, -9.57 to -2.52$)$ for timed-walk responders and 0.85 (95\% CI, -0.72 to 2.43 ) for nonresponders $(P<0.001)$.
The populations of the three clinical trials were generally similar among the studies, enabling pooling of populations to increase the discriminative power of the data and facilitate evaluation of responses based on demographic and clinical characteristics. ${ }^{66,67}$ The pooled population consisted of 631 patients from the modified intent-to-treat (mITT) analysis populations from MS-F202, MS-F203, and MS-F204; 237 were randomized to placebo and 394 to dalfampridine-ER $10 \mathrm{mg}$. The mITT population was defined prospectively in each study as all randomized patients who received the double-blind investigational drug and who had had at least one efficacy assessment during the double-blind treatment period. Only patients in the placebo and dalfampridine-ER $10 \mathrm{mg}$ groups from the MS-F202 study were used in the pooled analysis. Discontinuations due to AEs were 3.7\% and $2.1 \%$ from the dalfampridine-ER and placebo groups, respectively; there were no discontinuations due to lack of efficacy.

In the pooled population, the proportion of dalfampridineER responders was significantly higher relative to placebo, $37.3 \%$ versus $8.9 \%(P<0.001)$, and these proportions were consistent with those observed in the individual clinical trials (Figure 1) ${ }^{66}$ Among the dalfampridine-ER responders, the average percent change in walking speed was $25.3 \%$, compared with $5.8 \%$ in the placebo group (Figure 2) ${ }^{66}$ The mean improvement in pooled walking speed observed with dalfampridine-ER consistently demonstrated clinical relevancy, since percent changes in the pooled populations were all greater than $20 \%$, as was the mean improvement in the individual studies.

In an analysis to evaluate response rates stratified by demographic characteristics, consistency of response was observed across the pooled population when stratified by gender, race, age, and body mass index, as indicated by the interaction $P$ values, which were all $>0.100$ (Figure 3) ${ }^{67}$ Similarly, response rates among patients treated with dalfampridine-ER were independent of disease course (interaction $P=0.554$ ) or duration (interaction $P=0.318$; Figure 4). When stratified by baseline EDSS scores of $\leq 5.5,=6, \geq 6.5$, the proportion of dalfampridine-ER responders was similar among all strata, 36.1\%, 35.7\%, and $39.6 \%{ }^{67}$ Neither the use of immunomodulatory drugs nor the type of immunomodulatory drug appeared to have an effect on the dalfampridine-ER responder rate. ${ }^{67}$ The dalfampridine-ER responder rate among users of such therapies was $36.0 \%$, and among nonusers was $39.8 \%$. For patients using interferon beta, glatiramer acetate, or natalizumab, the dalfampridine-ER response rates were 


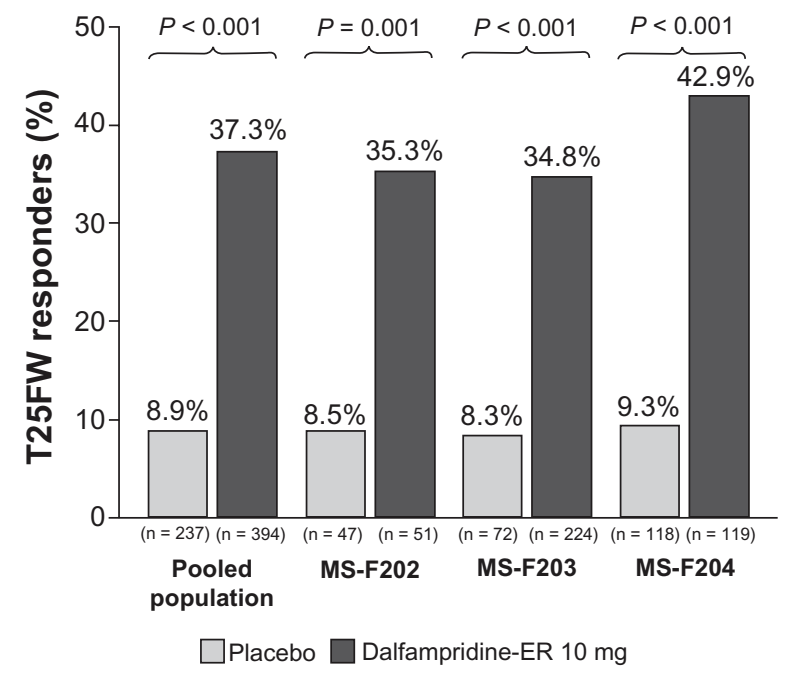

Figure I Proportion of timed 25-foot walk (T25FW) responders in the pooled analysis and component studies (modified intent-to-treat population). ${ }^{67}$

Note: Efficacy comparisons between placebo and dalfampridine-ER were performed using analysis of variance models with main effects for treatment group and center in the individual studies, main effects for treatment group, study, and center in the pooled analysis.

Abbreviation: MS, multiple sclerosis; ER, extended release.

$36.8 \%, 37.1 \%$, and $27.3 \%$, respectively. These results suggest that dalfampridine-ER may be of potential benefit to patients regardless of whether or not they are using immunomodulatory therapies.

Baseline walking speed, stratified by quartiles computed for each study and treatment group, did not have an effect on responder rate, and ranged from $31.3 \%-40.4 \%$ and $5.1 \%-10.3 \%$ for dalfampridine-ER and placebo, respectively, across quartiles (interaction $P=0.930$ ). Across all patients treated with dalfampridine-ER regardless of whether they were responders, baseline walking speed had no effect on the average percent improvement in walking speed, which ranged from $12.8 \%$ to $13.9 \%$ across quartiles, and was greater than the $3.0 \%$ to $9.1 \%$ improvement among placebo patients. ${ }^{66}$

\section{Open-label extensions of MS-F203 and MS-F204}

As part of the clinical development program, long-term extension (LTE) studies were initiated to evaluate the longterm safety, tolerability, and efficacy of dalfampridine-ER subsequent to the termination of the two Phase III pivotal trials. ${ }^{68}$ MS-F203EXT was the open-label, extension study of MS-F203, and MS-F204EXT was the corresponding open-label extension study for patients who participated in MS-F204. All patients who enrolled in the LTE received open-label dalfampridine-ER $10 \mathrm{mg}$ twice daily, and efficacy was assessed as the average percent change in walking speed from the double-blind study baseline, defined as the average of four pretreatment visits. However, since the parent studies required a safety assessment 2 weeks after the last doubleblind dose, patients had a minimum 2-week period without therapy prior to re-initiation of treatment.

Patients treated with dalfampridine-ER in the parent study were stratified based on response category during double-blind treatment, ie, responders or nonresponders. Among 224 patients in the MS-F203 dalfampridine-ER group with at least one double-blind T25FW assessment, 197 (70 dalfampridine-ER responders and 127 nonresponders) entered MS-F203EXT and completed at least one T25FW; of 119 comparable patients in MS-F204, 109 entered MS-F204EXT (49 dalfampridine-ER responders and 60 nonresponders) and completed at least one T25FW assessment. At termination of the LTEs, although

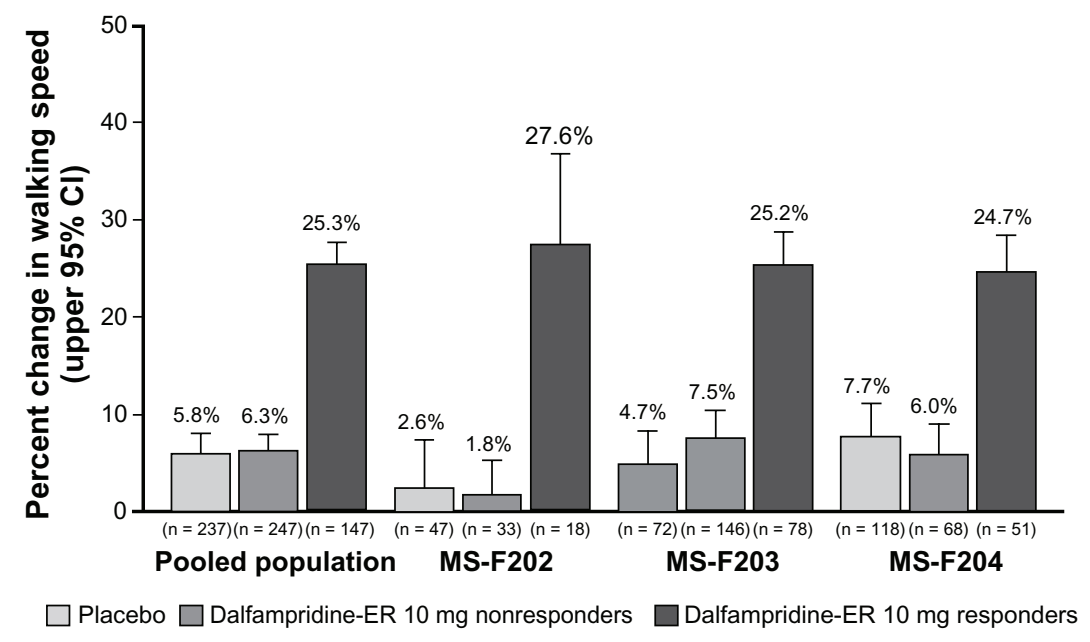

Figure 2 Average percent change from baseline during the treatment period in walking speed among dalfampridine extended release (ER) responders relative to placebo group and dalfampridine-ER nonresponders in the pooled analysis and component studies (modified intent-to-treat population). ${ }^{66}$ Abbreviation: MS, multiple sclerosis; ER, extended release. 

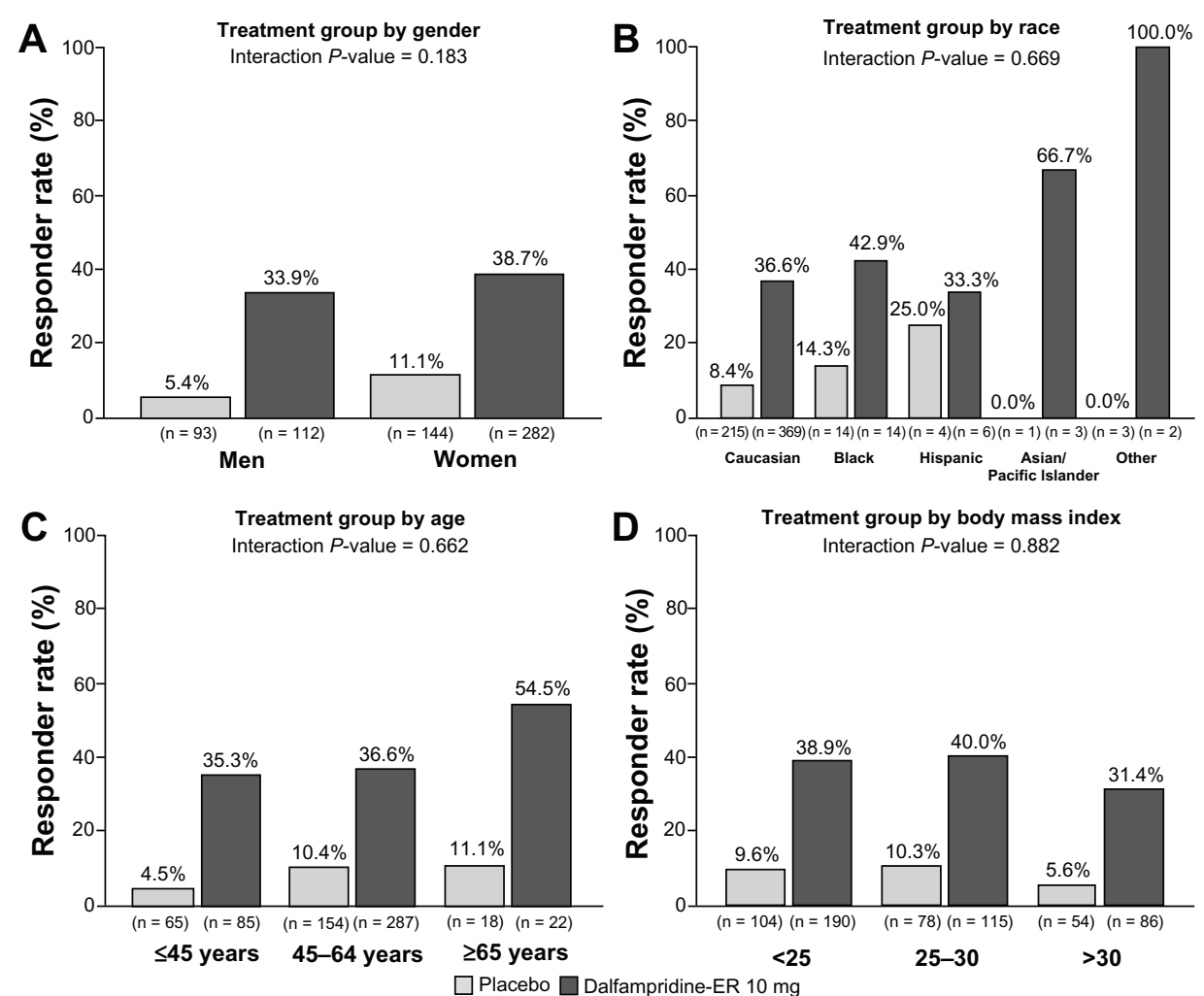

Figure 3 Pooled analysis of timed-walk responder rate stratified by baseline demographic characteristics (modified intent-to-treat population) ${ }^{67}$ (A) Gender. (B) Race. (C) Age. (D) Body mass index.

Abbreviation: ER, extended release.

the number of patients remaining was low, these patients had maximum exposure of up to 5 years in MS-F203EXT (Figure 5A) and 2.8 years for MS-F204EXT (Figure 5B) ${ }^{68}$

As expected, improvements in walking speed achieved during the double-blind period of both parent studies were lost after dalfampridine-ER discontinuation. However, re-initiation of dalfampridine-ER in the extension studies subsequently resulted in recovery of improvements by the initial 2-week LTE assessment. ${ }^{68}$ This recovery of walking speeds was similar to that observed at the end of the double-blind treatment period (Figure 5). Over the duration of both LTEs, walking speed remained improved among responders relative to nonresponders at the majority of protocol-defined assessment visits, and patient retention was greater among the responders. It is likely that the increased walking speed at later time points may be accounted for by the self-selection process that is characteristic of most LTEs; patients who achieved benefits and maintained tolerability tended to remain in the study for a longer duration than those who did not. Figure 6 clarifies the separation between responders and nonresponders when the issue of dropouts is eliminated by presenting walking-speed data only for those patients with continuous participation for approximately
2 years. These data are consistent with Figure 5 in showing that there is a gradual slowing of walking speed over time that is likely to reflect disease progression rather than loss of treatment benefit; similar rates of reduction in walking speed over time were previously observed in an analysis of secondary progressive MS subjects treated for 2 years with placebo or interferon $\beta$-1a in a clinical trial. ${ }^{69}$ Nevertheless, the data from both LTEs indicate that the subset of responders was able to maintain long-term improvements in walking speed relative to the nonresponder group at most visits.

\section{Post hoc analyses of changes in MSWS- 12}

In both Phase III clinical trials, T25FW responders had significantly greater improvements in the patient-reported MSWS-12 than nonresponders, regardless of treatment assignment. A post hoc analysis was performed to determine whether the observed changes in MSWS-12 among dalfampridine-ER responders were clinically meaningful, since statistically significant changes may not necessarily be clinically relevant. While the estimated change from baseline in MSWS-12 among dalfampridine-ER responders, -6.61 , was statistically significant $(P<0.001)$, both the 7.3-point difference between dalfampridine-ER responders and 

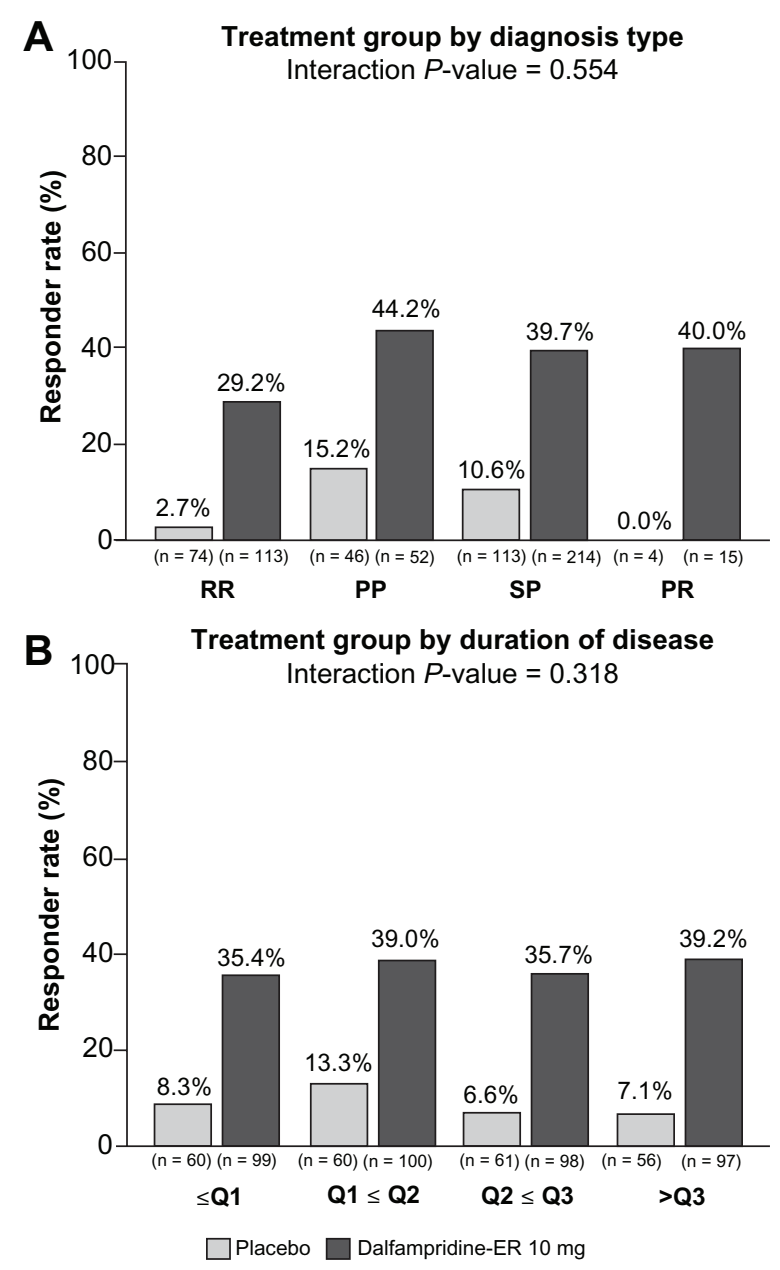

Figure 4 Pooled analysis of timed-walk responder rate stratified by multiple sclerosis (MS) disease characteristics (modified intent-to-treat population) ${ }^{67}$ (A) MS type. Relapsing remitting (RR), secondary progressive (SP), primary progressive (PP), progressive relapsing (PR). Because of the low number of patients with progressiverelapsing $M S$, the analysis of treatment by MS disease course type was computed post hoc, using only the RR, PP, and SP patients. (B) MS duration stratified by quartiles: $\mathrm{Q} 1, \geq 0.1$ to $<6.2$ years; $\mathrm{Q} 2, \geq 6.2$ to $<10.8$ years; $\mathrm{Q} 3, \geq 10.8$ to $<15.5$ years; $\mathrm{Q} 4, \geq 15.5$ years to 45.6 years (maximum).

Abbreviation: $E R$, extended release.

placebo and the 7.02-point difference with all nonresponders were clinically significant. ${ }^{70}$

Based on the Initiative on Methods, Measurement, and Pain Assessment in Clinical Trials (IMMPACT) recommendations that a change score is clinically meaningful when there is evidence from at least two of four methods, ${ }^{71}$ all four methods were used to evaluate the change in MSWS-12 scores: anchorbased for within-subject studies, anchor-based for betweensubject studies, distribution-based for within-subject studies, and distribution-based for between-subject studies. Using data from the two Phase III clinical trials and five other studies that incorporated the MSWS-12, all four methods demonstrated that the mean change in MSWS-12 score associated with dalfampridine-ER responders represented a clinically significant

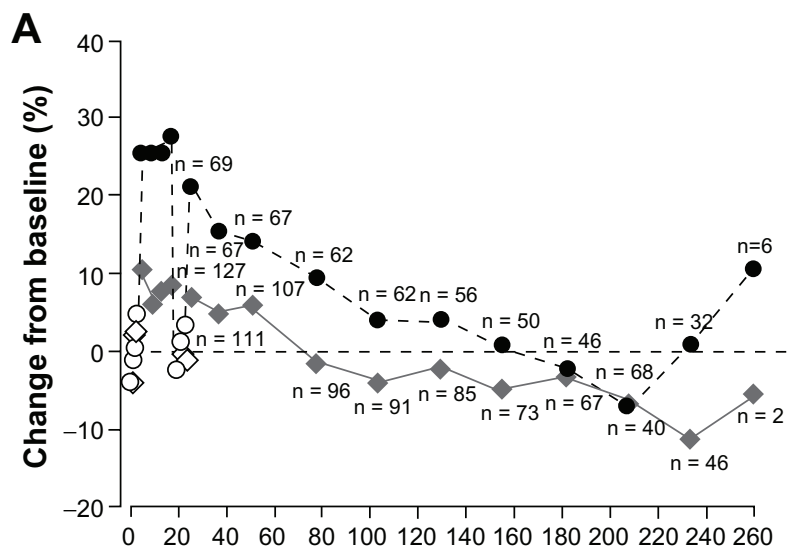

Scheduled time point (weeks)

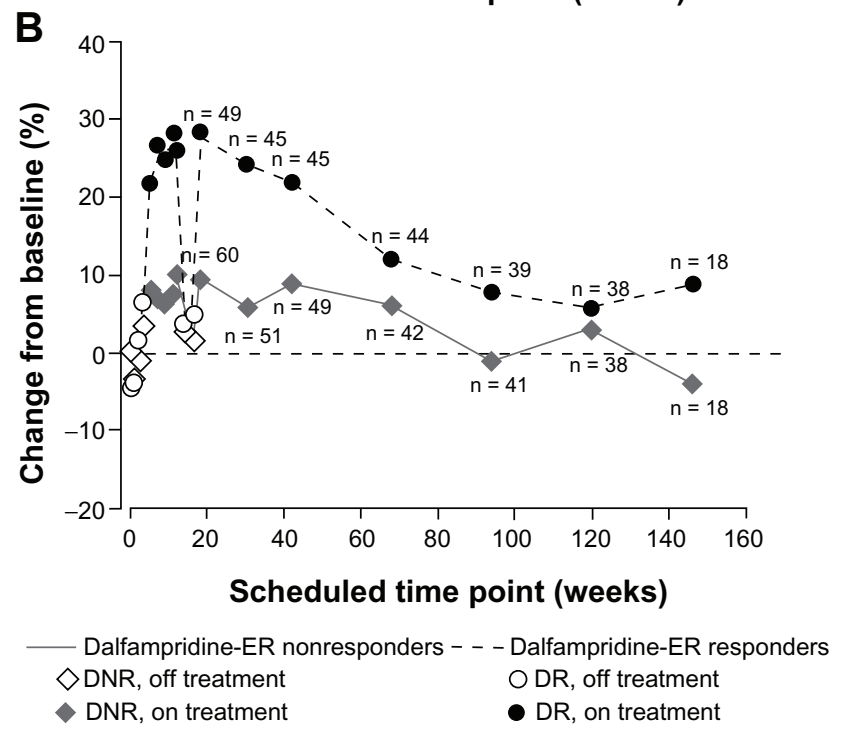

Figure 5 Percent change from baseline in walking speed in the parent studies and open-label long-term extensions by double-blind dalfampridine extended release (ER) responder status. (A) MS-F203. (B) MS-F204.

Abbreviations: DNR, dalfampridine-ER nonresponders; DR, dalfampridine-ER responders.

change..$^{70}$ Importantly, the two estimates of the minimum clinically important difference from the patient's perspective for change in the MSWS-12, 4.0 points (6.23-2.19) for individuals who reported mild improvement and 5.7 points $(-3.47$ to +2.19$)$ for those mildly worse, were both exceeded by the observed changes among dalfampridine-ER responders. In contrast, the estimated changes from baseline among nonresponders ( 0.32 points) and placebo-treated patients $(-0.69$ points $)$ in the clinical trials were neither statistically nor clinically significant. ${ }^{70}$

Further analysis of item-level scores showed that dalfampridine-ER responders demonstrated statistically significant improvements on eleven of the twelve MSWS-12 item scores, compared with nonresponders. The single item that was not statistically significant, "ability to run," was characterized by a floor effect that limited the ability to detect a change. ${ }^{72}$ 

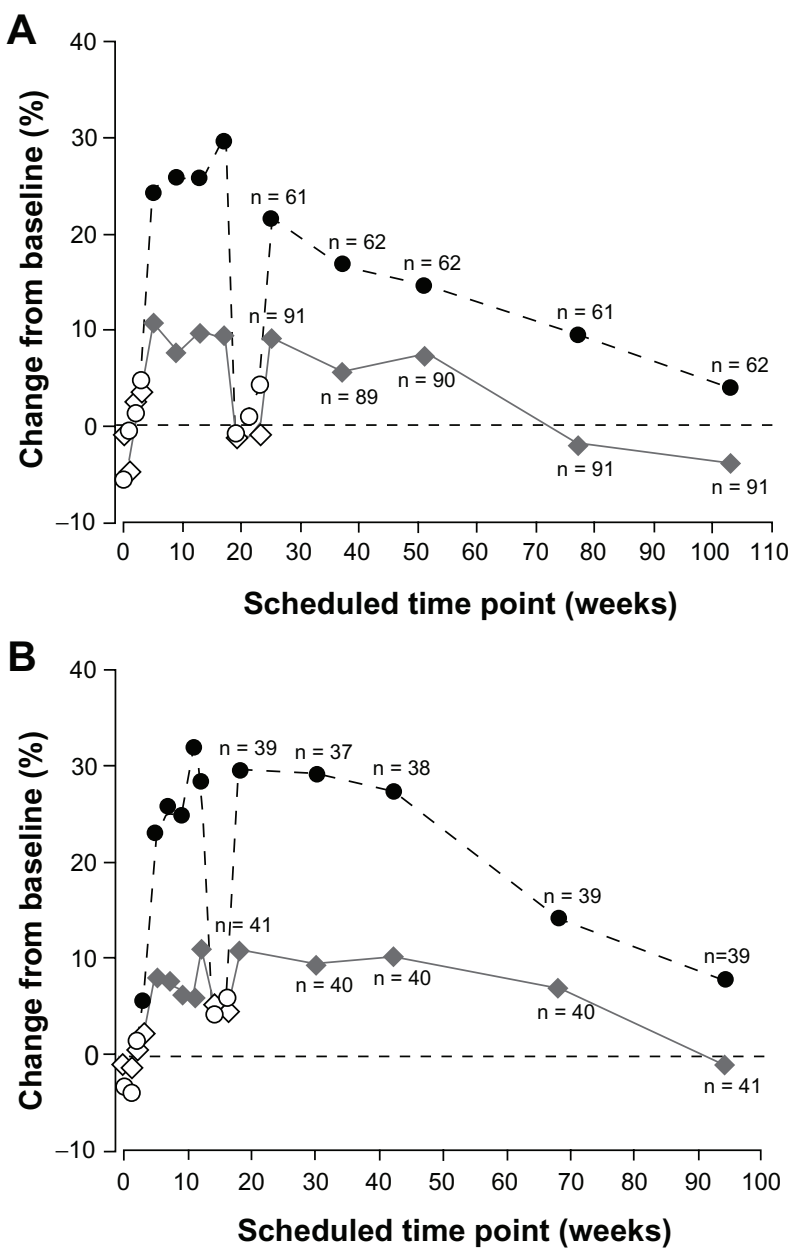

$\begin{array}{ll}\text { - Dalfampridine-ER nonresponders }- & - \text { Dalfampridine-ER responders } \\ \diamond D N R, \text { off treatment } & \text { O DR, off treatment } \\ \diamond D N R, \text { on treatment } & -D R, \text { on treatment }\end{array}$

Figure 6 Percent change from baseline in walking speed by double-blind dalfampridineER responder status among those patients with continuous participation. (A) At week 104 in MS-F203. (B) At week 96 in MS-F204.

Abbreviations: ER, extended release; DNR, dalfampridine-ER nonresponders; $D R$, dalfampridine-ER responders.

\section{Tolerability profile}

The safety populations of the individual trials consisted of all patients who received at least one dose of the study drug, and included the off-drug and washout periods, in addition to the double-blind treatment period. Dalfampridine-ER was generally well tolerated, and the safety profile was similar in all the individual trials. In the Phase II, doseranging study, the incidence of treatment-emergent adverse events (TEAEs) was $87 \%$ among the dalfampridine-ER $10 \mathrm{mg}$ group, compared with $81 \%$ of placebo patients. ${ }^{62}$ The majority of these TEAEs were of mild or moderate severity, $80 \%$ and $82 \%$ in the dalfampridine-ER $10 \mathrm{mg}$ and placebo groups, respectively; there were no serious TEAEs in the dalfampridine-ER $10 \mathrm{mg}$ group, and two in the placebo group. TEAEs were observed at higher frequency in the $15 \mathrm{mg}$ and $20 \mathrm{mg}$ dose groups, particularly with respect to central nervous system-related AEs, such as balance problems, paresthesias, dizziness, and insomnia. ${ }^{62}$ Although two seizures occurred in this trial, these appeared to be dose-related; and both occurred in patients who received dalfampridine-ER $20 \mathrm{mg}$, one of which involved accidental overdose. ${ }^{62}$

The overall incidence of TEAEs was similar among patients treated with dalfampridine-ER in both Phase III trials, $84 \%$ in MS-F203 and $86 \%$ in MS-F204, ${ }^{49,50}$ TEAEs in the placebo group were $81 \%$ and $66 \%$ in the two trials, respectively. Most TEAEs were generally of mild or moderate severity; serious TEAEs were reported by $7 \%$ of dalfampridine-ER patients in MS-F203 (none in the placebo group) ${ }^{49}$ and by $2.5 \%$ of placebo patients and $4.2 \%$ of dalfampridine-ER patients in MS-F204. ${ }^{50}$ Serious events occurred in a wide variety of systems and classes and were mostly considered unrelated to treatment. There were two reported seizure events: one, in a dalfampridine-ER-treated patient, was observed as a focal seizure during an episode of severe sepsis, and the other was reported as a complex partial seizure in a patient treated with placebo.

In the pooled safety population from the Phase II (placebo and dalfampridine-ER $10 \mathrm{mg}$ dose) trial and two Phase III trials, dalfampridine-ER demonstrated an overall favorable safety and tolerability profile that was consistent with the individual studies. TEAEs were reported in $84.8 \%$ of pooled patients in the dalfampridine-ER group, compared with $73.5 \%$ with placebo (Table 2). Serious TEAEs occurred in $22(5.5 \%)$ patients in the dalfampridine-ER $10 \mathrm{mg}$ group, and five $(2.1 \%)$ patients in the placebo group, and among the dalfampridine-ER patients, the seizure rate was $0.25 \%$. The most common TEAEs were consistent with those reported in the individual trials, and they and appear to be related to increases in central nervous system excitation. Of the most common TEAEs, reported in $\geq 5 \%$ of the pooled dalfampridine-ER-treated population, those with the greatest difference between dalfampridine-ER and placebo included urinary tract infections, insomnia, nausea, and balance disorder (Table 2) ${ }^{67}$

The safety and tolerability profile observed in the LTEs was consistent with the double-blind phase of the parent trials; the most common adverse events were urinary tract infections, falls, MS relapses, arthralgia, and peripheral edema. ${ }^{68} \mathrm{~A}$ total of four seizure-related adverse events were reported during the extension studies among the patients who were treated with dalfampridine-ER in the parent trial (1.5\%); 
Table 2 Treatment-emergent adverse events (TEAEs) that occurred in $\geq 5 \%$ of dalfampridine extended release (ER)-treated patients in the pooled MS-F202, MS-F203, and MS-F204 safety population ${ }^{\mathrm{a}}$

\begin{tabular}{lll}
\hline TEAEs & Incidence, $\mathbf{n}(\%)$ & \\
\cline { 2 - 3 } & $\begin{array}{l}\text { Dalfampridine-ER I } \mathbf{~} \mathbf{~ m g} \\
\text { twice daily }(\mathbf{n}=\mathbf{4 0 0})\end{array}$ & $\begin{array}{l}\text { Placebo } \\
\mathbf{( n = 2 3 8 )}\end{array}$ \\
\hline Any TEAE & $339(84.8)$ & $175(73.5)$ \\
Serious TEAE & $22(5.5)$ & $5(2.1)$ \\
Fall & $64(16.0)$ & $39(16.4)$ \\
Urinary tract infection & $58(14.5)$ & $22(9.2)$ \\
Insomnia & $37(9.3)$ & $9(3.8)$ \\
Asthenia & $33(8.3)$ & $10(4.2)$ \\
Dizziness & $31(7.8)$ & $10(4.2)$ \\
Headache & $30(7.5)$ & $10(4.2)$ \\
Nausea & $28(7.0)$ & $6(2.5)$ \\
Fatigue & $26(6.5)$ & $11(4.6)$ \\
Balance disorder & $23(5.8)$ & $3(1.3)$ \\
Upper respiratory & $23(5.8)$ & $17(7.1)$ \\
tract infection & & \\
MS relapse ${ }^{b}$ & $21(5.3)$ & $9(3.8)$ \\
Back pain & $22(5.5)$ & $5(2.1)$ \\
\hline
\end{tabular}

Notes: Includes off-drug, washout periods; 'bsimilar incidences in drug and placebo groups during active treatment.

Abbreviation: MS, multiple sclerosis.

none of the patients treated with placebo in the parent trial reported a seizure event during the extension. An additional patient who was enrolled in MS-F203EXT had a grand mal convulsion that occurred approximately 1 month after stopping the study drug.

Dalfampridine $10 \mathrm{mg}$ extended release tablets have been available in the US market since March 2010, and safety experience has been available from clinical practice through March 2011, encompassing the exposure of approximately 46,200 patients and representing approximately 14,500 patient-years.

Descriptive analysis was performed to provide information on all postmarketing AEs that were spontaneously reported. ${ }^{73}$ The most frequently reported AEs, with a prevalence of $\geq 2 \%$ of all reported cases and reported as the percent of total AEs, included dizziness (5.7\%), insomnia (4.5\%), balance disorder (3.9\%), headache (3.2\%), nausea $(2.8 \%)$, urinary tract infection $(2.4 \%)$, asthenia $(2.0 \%)$, and back pain (2.0\%). These were also the most common AEs reported during clinical development, and all are included in the current US product label. ${ }^{28}$ Additional new findings were related to lack of efficacy (4.5\%) - which may be expected, considering that dalfampridine-ER has been demonstrated to be effective in approximately $37 \%$ of patients in clinical trials - and to inappropriate dosing (5.0\%).

A total of 85 seizures were reported during postmarketing surveillance, representing an incidence rate of approximately
5.9/1000 patient-years of use. Of these seizures, 82 were either reported or confirmed by a health care practitioner, and an additional three cases were reported directly by patients but were not confirmed by a health care practitioner. Beyond the risk of seizure that is inherent in the MS population which has been suggested to be threefold higher than that of the general population, ${ }^{74,75}$ with the best estimate of a first seizure incidence reported as 3.49/1000 patient-years (95\% $\mathrm{CI}=1.96-5.02 / 1000$ patient-years $)^{76}-61 \%$ of the patients with seizures had additional potential risk factors for seizure. These risk factors included use of concurrent medications with a labeled seizure risk $(n=45)$, incorrect dosing $(n=5)$, a history of convulsions $(\mathrm{n}=5)$, renal impairment $(\mathrm{n}=3)$, and prior head injury $(\mathrm{n}=1) ; 54 \%$ of patients had one risk factor, and $7 \%$ had two or three risk factors. ${ }^{73}$ Duration of treatment prior to the event ranged from one dose to 365 days, and 23 of the 82 confirmed cases $(28 \%)$ occurred within a week of starting dalfampridine-ER.

\section{Clinical relevance}

While walking impairment is among the most visible and disabling manifestations of MS, it has until recently been under-recognized as a therapeutic target for pharmacologic intervention. The approval of dalfampridine-ER fills the unmet need for a pharmacologic therapy targeting this disability. This drug is now among those therapies available for the treatment of symptoms affecting the daily function and activities of patients with MS, many of which may be overlooked during treatment with immunomodulatory drugs, which are the mainstay of MS management.

Dalfampridine-ER appears to significantly improve walking speed and leg motor function in about $37 \%$ of people with walking disability due to MS, with an overall increase of approximately $25 \%$ in walking speed that is clinically relevant from the patient's perspective. The objective improvements in walking speed observed with the T25FW were additionally shown to be clinically meaningful to patients, as measured by the MSWS-12. Responsiveness to treatment appears to be independent of baseline walking speed, age, type, and duration of MS, EDSS disability score, and use of immunomodulatory drugs, and thus dalfampridine-ER can be used in patients across the range of demographic and disease characteristics found in clinical practice. Since walking impairment is present even in patients with less obvious levels of disability and with recent onset of MS, these patients may also benefit from early treatment.

Dalfampridine-ER is generally well tolerated. Spontaneous safety data emerging from the US postmarketing experience 
are consistent in terms of the safety-profile types of events seen during clinical development, and no new safety signals were reported. Dizziness, insomnia, balance disorder, headache, nausea, urinary tract infection, asthenia, and back pain were the most frequently reported AEs in clinical trials and postmarketing surveillance. While the first-year seizure incidence reported from the postmarketing data was not substantially different from that observed in clinical trials, there is a dose-dependent increase in the occurrence of seizures at doses higher than the recommended dose of $10 \mathrm{mg}$ twice daily. It is thus especially important to adhere to the dosing in the approved label, and to ensure that other forms of 4-AP, such as those provided by compounding pharmacies, are not used in conjunction with dalfampridine-ER.

\section{Conclusion}

Walking impairment has an adverse impact on patients' lives that is manifested in a variety of daily activities. Treatment with dalfampridine-ER has been shown in clinical studies to improve walking speed in about one third of MS patients, regardless of MS type or duration, baseline walking speed, or demographic characteristics. The increase in walking speed was paralleled by patient-reported improvements in walking impairment. Data from long-term, open-label extension studies and spontaneous postmarketing safety data confirm the safety profile observed during clinical development of dalfampridine-ER.

\section{Disclosure}

Editorial assistance in preparation of this manuscript was provided by the Curry Rockefeller Group, LLC. Editorial assistance was funded by Acorda Therapeutics, Inc. HRH and ARB are employees of Acorda Therapeutics, Inc, and hold stock in the company.

\section{References}

1. National Multiple Sclerosis Society. FAQs about MS [webpage on the Internet]. New York: National Multiple Sclerosis Society; nd. Available from: http://www.nationalmssociety.org/about-multiple-sclerosis/what-weknow-about-ms/faqs-about-ms/index.aspx. Accessed January 11, 2012.

2. Whetten-Goldstein K, Sloan FA, Goldstein LB, Kulas ED. A comprehensive assessment of the cost of multiple sclerosis in the United States. Mult Scler. 1998;4(5):419-425.

3. Kobelt G, Berg J, Atherly D, Hadjimichael O. Costs and quality of life in multiple sclerosis. A cross-sectional study in the United States. Neurology. 2006;66:1696-1702.

4. Kobelt G, Berg J, Lindgren P, Fredrikson S, Jonsson B. Costs and quality of life of patients with multiple sclerosis in Europe. J Neurol Neurosurg Psychiatry. 2006;77:918-926.

5. Salter AR, Cutter GR, Tyry T, Marrie RA, Vollmer T. Impact of loss of mobility on instrumental activities of daily living and socioeconomic status in patients with MS. Curr Med Res Opin. 2010;26(2):493-500.
6. Figved N, Myhr KM, Larsen JP, Aarsland D. Caregiver burden in multiple sclerosis: the impact of neuropsychiatric symptoms. J Neurol Neurosurg Psychiatry. 2007;78(10):1097-1102.

7. Dunn J. Impact of mobility impairment on the burden of caregiving in individuals with multiple sclerosis. Expert Rev Pharmacoecon Outcomes Res. 2010;10(4):433-440.

8. Buchanan R, Huang C. Health-related quality of life among informal caregivers assisting people with multiple sclerosis. Disabil Rehabil. 2011;33(2):113-121.

9. Waxman SG. Demyelinating diseases - new pathological insights, new therapeutic targets [editorial]. N Engl J Med. 1998;338(5):323-325.

10. Smith KJ, McDonald WI. The pathophysiology of multiple sclerosis: the mechanisms underlying the production of symptoms and the natural history of the disease. Philos Trans R Soc Lond B Biol Sci. 1999; 354(1390):1649-1673.

11. Dutta R, Trapp BD. Pathogenesis of axonal and neuronal damage in multiple sclerosis. Neurology. 2007;68(22 Suppl 3):S22-S31.

12. McDonald WI. Pathophysiology in multiple sclerosis. Brain. 1974; 97(1):179-196.

13. Compston A, Coles A. Multiple sclerosis. Lancet. 2008;372(9648): $1502-1517$.

14. Kurtzke JF. Rating neurologic impairment in multiple sclerosis: an expanded disability status scale (EDSS). Neurology. 1983;33(11): 1444-1452.

15. Rudick RA, Cutter G, Baier M, et al. Use of the multiple sclerosis functional composite to predict disability in relapsing MS. Neurology. 2001;56:1324-1330.

16. Sutliff M. Contribution of impaired mobility to patient burden in multiple sclerosis. Curr Med Res Opin. 2010;26(1):109-119.

17. Yildiz M. Slower walking speed limits the activities of daily living of multiple sclerosis patients [abstract]. J Neurol. 2011;258:96.

18. LaRocca N. Impact of walking impairment in multiple sclerosis: perspectives of patients and care partners. Patient. 2011;4(3): 189-201.

19. Heesen C, Bohm J, Reich C, Kasper J, Goebel M, Gold SM. Patient perception of bodily functions in multiple sclerosis: gait and visual function are the most valuable. Mult Scler. 2008;14(7):988-991.

20. Myhr KM, Riise T, Vedeler C, Nortvedt MW, Grønning R, Nyland HI. Disability and prognosis in multiple sclerosis: demographic and clinical variables important for the ability to walk and awarding of disability pension. Mult Scler. 2001;7(1):59-65.

21. Martin CL, Phillips BA, Kilpatrick TJ, et al. Gait and balance impairment in early multiple sclerosis in the absence of clinical disability. Mult Scler. 2006;12(5):620-628.

22. Goldman M, Ann Marrie R, Cohen JA. Evaluation of the six-minute walk in multiple sclerosis subjects and healthy controls. Mult Scler. 2008; 14:383-390.

23. van Asch P. Impact of mobility impairment in multiple sclerosis $2-$ patients' perspectives. European Neurological Review. 2011;6(2): $115-120$.

24. Ben-Zacharia AB. Therapeutics for multiple sclerosis symptoms. $M t$ Sinai J Med. 2011;78(2):176-191.

25. de Sa JC, Airas L, Bartholome E, et al. Symptomatic therapy in multiple sclerosis: a review for a multimodal approach in clinical practice. Ther Adv Neurol Disord. 2011;4(3):139-168.

26. Frohman TC, Castro W, Shah A, et al. Symptomatic therapy in multiple sclerosis. Ther Adv Neurol Disord. 2011;4(2):83-98.

27. Samkoff LM, Goodman AD. Symptomatic management in multiple sclerosis. Neurol Clin. 2011;29(2):449-463.

28. Ampyra ${ }^{\circledR}$ (dalfampridine) extended release tablets [prescribing information]. New York: Acorda Therapeutics, Inc; 2010.

29. Therapeutic Goods Administration, Department of Health and Aging, Australian Government. Australian Public Assessment Report for Fampridine. Woden: Therapeutic Goods Administration; 2011. Available from: http://www.tga.gov.au/pdf/auspar/auspar-fampyra.pdf. Accessed January 11, 2012. 
30. European Medicines Agency Committee for Medicinal Products for Human Use. Summary of Opinion (Initial Authorisation): Fampyra; Fampridine. London: European Medicines Agency; 2011. Available from: http://www.ema.europa.eu/docs/en_GB/document_library/ Summary_of_opinion_-_Initial_authorisation/human/002097/ WC500106531.pdf. Accessed January 11, 2012.

31. Dunn J, Blight A. Dalfampridine: a brief review of its mechanism of action and efficacy as a treatment to improve walking in patients with multiple sclerosis. Curr Med Res Opin. 2011;27(7):1415-1423.

32. Targ EF, Kocsis JD. 4-Aminopyridine leads to restoration of conduction in demyelinated rat sciatic nerve. Brain Res. 1985;328(2):358-361.

33. Shi R, Blight AR. Differential effects of low and high concentrations of 4-aminopyridine on axonal conduction in normal and injured spinal cord. Neuroscience. 1997;77(2):553-562.

34. Stefoski D, Davis FA, Faut M, Schauf CL. 4-Aminopyridine improves clinical signs in multiple sclerosis. Ann Neurol. 1987;21(1):71-77.

35. Davis FA, Stefoski D, Rush J. Orally administered 4-aminopyridine improves clinical signs in multiple sclerosis. Ann Neurol. 1990;27(2): 186-192.

36. Van Diemen HA, Polman CH, van Dongen TM, et al. The effect of 4-aminopyridine on clinical signs in multiple sclerosis: a randomized, placebo-controlled, double-blind, cross-over study. Ann Neurol. 1992; 32(2):123-130.

37. Schwid SR, Petrie MD, McDermott MP, Tierney DS, Mason DH, Goodman AD. Quantitative assessment of sustained-release 4-aminopyridine for symptomatic treatment of multiple sclerosis. Neurology. 1997;48(4):817-821.

38. Blight AR, Henney HR III. Pharmacokinetics of 14C-radioactivity after oral intake of a single dose of 14C-labeled fampridine (4-aminopyridine) in healthy volunteers. Clin Ther. 2009;31(2):328-335.

39. Van Diemen HA, Polman CH, Koetsier JC, van Loenen AC, Nauta JJ, Bertelsmann FW. 4-Aminopyridine in patients with multiple sclerosis: dosage and serum level related to efficacy and safety. Clin Neuropharmacol. 1993;16(3):195-204.

40. Bever CT Jr, Young D, Anderson PA, et al. The effects of 4-aminopyridine in multiple sclerosis patients: results of a randomized, placebo-controlled, double-blind, concentration-controlled, crossover trial. Neurology. 1994; 44(6):1054-1059.

41. Hayes KC, Katz MA, Devane JG, et al. Pharmacokinetics of an immediate-release oral formulation of Fampridine (4-aminopyridine) in normal subjects and patients with spinal cord injury. J Clin Pharmacol. 2003;43(4):379-385.

42. Smith W, Swan S, Marbury T, Henney HR III. Single-dose pharmacokinetics of sustained-release fampridine (Fampridine-SR) in healthy volunteers and adults with renal impairment. J Clin Pharmacol. 2010; 50(2):151-159.

43. Vollmer T, Henney HR III. Pharmacokinetics and tolerability of single escalating doses of fampridine sustained-release tablets in patients with multiple sclerosis: a phase I-II, open-label trial. Clin Ther. 2009;31(10): 2206-2214.

44. March B, Cardi T. Assessment of the cardiac safety of fampridine-SR sustained-release tablets in a thorough QT/QTc evaluation at therapeutic and supratherapeutic doses in healthy individuals. Expert Opin Investig Drugs. 2009;18(12):1807-1815.

45. Henney HR III, Blight A, Faust B. Effect of food on the single-dose pharmacokinetics and tolerability of dalfampridine extended-release tablets in healthy volunteers. Am J Health Syst Pharm. 2011;68: 2148-2154.

46. Claxton AJ, Cramer J, Pierce C. A systematic review of the associations between dose regimens and medication compliance. Clin Ther. 2001; 23(8):1296-1310.

47. Saini SD, Schoenfeld P, Kaulback K, Dubinsky MC. Effect of medication dosing frequency on adherence in chronic diseases. Am J Manag Care. 2009; 15(6):e22-e33.

48. Richter A, Anton SE, Koch P, Dennett SL. The impact of reducing dose frequency on health outcomes. Clin Ther. 2003;25(8):2307-2335.
49. Goodman AD, Brown TR, Krupp L, et al. Sustained release of oral fampridine in multiple sclerosis: a randomised, double-blind, controlled trial. Lancet. 2009;373(9665):732-738.

50. Goodman AD, Brown TR, Edwards KR, et al. A phase 3 trial of extended release oral dalfampridine in multiple sclerosis. Ann Neurol. 2010;68(4):494-502.

51. Rudick RA, Cutter G, Reingold S. The multiple sclerosis functional composite: a new clinical outcome measure for multiple sclerosis clinical trials. Mult Scler. 2002;8:359-365.

52. Hobart JC, Riazi A, Lamping DL, Fitzpatrick R, Thompson AJ. Measuring the impact of MS on walking ability: the 12-Item MS Walking Scale (MSWS-12). Neurology. 2003;60(1):31-36.

53. Bethoux FA, Bennett SE. Evaluating walking mobility in patients with multiple sclerosis: utility of instruments in clinical trials and clinical practice. Int J MS Care. 2011;13(1):4-14.

54. Créange A, Serre I, Levasseur M, et al. Walking capacities in multiple sclerosis measured by global positioning system odometer. Mult Scler. 2007;13(2):220-223.

55. Cutter GR, Baier ML, Rudick RA, et al. Development of a multiple sclerosis functional composite as a clinical trial outcome measure. Brain. 1999;122(Pt 5):871-882.

56. Cohen JA, Fischer JS, Bolibrush DM, et al. Intrarater and interrater reliability of the MS functional composite outcome measure. Neurology. 2000;54(4):802-806.

57. Kalkers NF, de Groot V, Lazeron RH, et al. MS functional composite: relation to disease phenotype and disability strata. Neurology. 2000; 54(6):1233-1239.

58. Fischer JS, Rudick RA, Cutter GR, Reingold SC. The Multiple Sclerosis Functional Composite Measure (MSFC): an integrated approach to MS clinical outcome assessment. National MS Society Clinical Outcomes Assessment Task Force. Mult Scler. 1999;5(4):244-250.

59. Kaufman M, Moyer D, Norton J. The significant change for the timed 25-foot walk in the multiple sclerosis functional composite. Mult Scler. 2000;6:286-290.

60. Hoogervorst EL, Kalkers NF, Cutter GR, Uitdehaag BM, Polman CH. The patient's perception of a (reliable) change in the multiple sclerosis functional composite. Mult Scler. 2004;10:55-60.

61. Gijbels D, Dalgas U, Romberg A, et al. Which walking capacity tests to use in multiple sclerosis? A multicentre study providing the basis for a core set. Mult Scler. 2011;18(3):364-371.

62. Goodman AD, Brown TR, Cohen JA, et al. Dose-comparison trial of sustained-release fampridine in multiple sclerosis. Neurology. 2008; 71(15):1134-1141.

63. Schwid SR, Petrie MD, McDermott MP, Tierney DS, Mason DH, Goodman AD. Quantitative assessment of sustained-release 4-aminopyridine for symptomatic treatment of multiple sclerosis. Neurology. 1997;48(4):817-821.

64. Schwid SR, Goodman AD, McDermott MP, Bever CF, Cook SD. Quantitative functional measures in MS: what is a reliable change? Neurology. 2002;58(8):1294-1296.

65. Kragt JJ, van der Linden FA, Nielsen JM, Uitdehaag BM, Polman CH. Clinical impact of $20 \%$ worsening on timed 25 -foot walk and 9-hole peg test in multiple sclerosis. Mult Scler. 2006;12(5):594-598.

66. Edwards K, Brown T, Schapiro R, et al. Dalfampridine extended release tablets improve walking speed across a wide range of baseline deficits: pooled data from three placebo-controlled studies in patients with multiple sclerosis [abstract]. Neurology. 2010;74(Suppl 2):A565.

67. Brown T, Schapiro R, Edwards K, et al. Response to treatment with dalfampridine extended release tablets in patients with multiple sclerosis is independent of baseline patient characteristics and concomitant immunomodulator therapy [abstract]. Neurology. 2010; 74(Suppl 2):A543.

68. Goodman A, on behalf of the MS-F203, MS-F204, and extension study investigators. Updated analysis of open-label extension studies of dalfampridine extended release tablets in multiple sclerosis [abstract]. Mult Scler. 2011;17(Suppl):S245-S246. 
69. Cadavid D, Tang Y, O’Neill G. Responsiveness of the Expanded Disability Status Scale (EDSS) to disease progression and therapeutic intervention in progressive forms of multiple sclerosis. Rev Neurol. 2010;51(6):321-329.

70. Hobart J. Prolonged-release fampridine for multiple sclerosis: was the effect on walking ability clinically significant? [abstract]. Mult Scler. 2010;16 Suppl:S72.

71. Dworkin RH, Turk DC, Wyrwich KW, et al. Interpreting the clinical importance of treatment outcomes in chronic pain clinical trials: IMMPACT recommendations. J Pain. 2008;9(2):105-121.

72. Hobart J. Responder or non-responder, that is the question. Was the responder definition used in the dalfampridine extended release studies clinically meaningful? [abstract]. Neurology. 2011;76(Suppl 4):A70.
73. Jara M, Henney HR III, Wessel T, Barker G, Kargman D. Dalfampridine extended-release tablets: one year of post-marketing safety experience in the United States [abstract]. Mult Scler. 2011;17(Suppl 10):S216.

74. Nicoletti A, Sofia V, Biondi R, et al. Epilepsy and multiple sclerosis in Sicily: a population-based study. Epilepsia. 2003;44(11):1445-1448.

75. Poser CM, Brinar VV. Epilepsy and multiple sclerosis. Epilepsy Behav. 2003;4(1):6-12.

76. Eriksson M, Ben-Menachem E, Andersen O. Epileptic seizures, cranial neuralgias and paroxysmal symptoms in remitting and progressive multiple sclerosis. Mult Scler. 2002;8(6):495-499.

\section{Publish your work in this journal}

Degenerative Neurological and Neuromuscular Disease is an international, peer-reviewed, open access journal focusing on research into degenerative neurological and neuromuscular disease, identification of therapeutic targets and the optimal use of preventative and integrated treatment interventions to achieve improved outcomes, enhanced survival and quality of life for the patient. The manuscript management system is completely online and includes a very quick and fair peer-review system. Visit http://www.dovepress.com/testimonials.php to read real quotes from published authors.

Submit your manuscript here: http://www.dovepress.com/degenerative-neurological-and-neuromuscular-disease-journal 